\title{
IVD.I2 - Standardization of the XMAP Luminex methodology for simultaneous detection and differentiation of Dengue serotypes and Chikungunya viruses
}

Mauro Jorge Cabral Castro ${ }^{1 \star}$; Larissa de Souza Santana ${ }^{1}$; Jorge Luiz dos Santos Gonçalves ${ }^{1}$; Eduardo Scarlatelli Pimenta ${ }^{2}$; Marta Guimarães Cavalcanti²; Regina Helena Saramago Peralta ${ }^{3}$; José Mauro Peralta ${ }^{1}$.

1UFRJ - Universidade Federal do Rio de Janeiro;

2UFRJ/ HUCCF (Hospital Univesitário Clementino Fraga Filho);

3UFF - Universidade Federal Fluminense.

Introduction: Arboviruses are among the most important emerging and reemerging virus in the world, transmitted mainly by the bite of the female mosquito of the genus Aedes. In Brazil, dengue virus (DENV) infection has been established since 1985, and more recently, Zika (ZIKV) and Chikungunya (CHIKV) virus infections have emerged as a public health concern. The clinical diagnosis of these arboviruses in the areas with DENV, CHIKV and ZIKV cocirculation become difficult due to the similarity of the signs and symptoms presented in these infections. Therefore, the search for a differential laboratory diagnosis is very important.

Objective: The objective of this study is to standardize a laboratory diagnostic method for the simultaneous detection of the viral RNA of the four DENV serotypes and CHIKV in a single reaction using the RT-PCR/ Luminex methodology.

Methodology: For this, we used control samples for CHIKV and DENV serotypes, in addition to 10 samples of individuals without symptoms of arbovirus infection and without cDNA amplification by PCR as negative controls. To do this, the RNA extraction from the samples was performed, followed by cDNA synthesis, RT-PCR amplification obtained biotinylated amplicons and detection by the Luminex ${ }^{\circledast}$ system. For the evaluation of the method, we used 10 samples with previous results by PCR of CHIKV and/or DENV. Biotinylated amplicons were used in the Luminex reaction step. In this step, we evaluated three different hybridization temperatures $\left(42^{\circ} \mathrm{C}, 45^{\circ} \mathrm{C}\right.$ and $\left.48^{\circ} \mathrm{C}\right)$.

Results: In a temperature of $42^{\circ} \mathrm{C}$ obtained satisfactory results, being able to differentiate the four serotypes of DENV and CHIKV evaluated. All 10 samples previously analyzed by PCR reacted in the Luminex system, confirming their simple infections, DENV or CHIKV, and cases of co-infection with CHIKV and DENV. The time to obtain results was lower than when the PCR was performed.

Conclusion: The RT-PCR/ Luminex ${ }^{\circledast}$ system designed in this study was able to detect and differentiate infections by DENV and CHIKV, in addition to detecting cases with co-infection between these viruses. However, a larger number of samples will be tested in the validation step, besides inclusion for detection of ZIKV. Therefore, this methodology can be an effective tool to aid in the simultaneous detection of these viruses, besides the possibility of adding new targets.

Keywords: Arboviruses; xMAP Luminex; molecular diagnosis 